\title{
0861 THE EFFECT OF EDUCATION AND SAFETY EQUIPMENT ON BURN AND SCALD PREVENTION PRACTICES AND ON CHILDHOOD THERMAL INJURIES: UPDATE OF A SYSTEMATIC REVIEW AND META-ANALYSIS
}

P Wynn*, N llyas, D Kendrick, A Sutton, C Coupland, C Mulvaney, M Watson Correspondence: Division of Primary Care, Room 1401, 14th Floor, Tower Building, University Park, Nottingham University, Nottingham NG7 2RD, UK

\subsection{6/ip.2010.029215.861}

Objective Childhood burns and scalds are a significant public health problem. This systematic review and meta-analysis evaluates whether home safety education and the provision of safety equipment increases burn and scald prevention practices and reduces thermal injuries.

Method A range of bibliographic databases were searched until April 2009. Search methods also included other electronic sources; hand searching of conference abstracts, IPJ and reference lists. We included randomised controlled trials (RCTs), nonRCTs, and controlled before and after studies (CBA), involving children and young people aged 0-19 years, and their families.

Results Preliminary results indicate that 49 studies met the inclusion criteria (29 RCTs, 6 non-RCTs, 13 CBAs and 1 CBA or non RCT). 27 studies were included in at least one metaanalysis. Families receiving home safety education + /- safety equipment were significantly more likely to have a functional smoke alarm (OR 1.83, 95\% CI 1.22 to 2.73) and to have safe hot-tap water temperatures (OR 1.51, 95\% CI 1.11 to 2.07). There was some evidence that interventions increased the prevalence of fireguards (OR 1.39, 95\% CI 1.00 to 1.94). The prevalence of fire extinguishers was increased only by interventions providing these (OR 4.67, 95\% CI 1.78 to 12.25 ).

Conclusions Home safety education and the provision of safety equipment are effective in improving the prevalence of functioning smoke alarms and safe hot tap water temperatures. They may be effective in increasing the prevalence of fireguards and fire extinguishers. Further work is required to explore the effect of improving safety practices on thermal injury occurrence. 